\title{
Impacto del ambiente en la salud escolar: representaciones sociales del equipo de salud.
}

\section{Impact of the environment on school students' health: social representations of the health care team}

\author{
Graciela Fabiana Scruzzi ${ }^{1}$, Constanza Rodríguez Junyent ${ }^{1}$, Catalina Tosini ${ }^{1}$, Candelaria Bertaina ${ }^{1}$, María \\ Carlina Bustos ${ }^{1}$.
}

1 Universidad Católica de Córdoba, Facultad de Ciencias de la Salud, Carrera de Nutrición

\section{Resumen}

INTRODUCCIÓN: La salud ambiental comprende aspectos de la salud humana que incluye la calidad de vida y es determinada por condiciones ambientales, físicas, sociales y alimentarias.

OBJETIVO: Indagar las representaciones sociales (RS) sobre el impacto del ambiente en la salud de los escolares en el equipo de salud (ES) de dos Centros de Atención Primaria de Salud municipales (CAPS), durante el ciclo 2019 en la ciudad de Córdoba, Argentina

DISEÑO METODOLÓGICO: La investigación se llevó a cabo desde la perspectiva cualitativa, basada en la teoría fundamentada, con diseño emergente. Se realizaron 13 entrevistas abiertas, a través del método de comparación constante. Los datos fueron validados por triangulación de investigadores.

RESULTADOS: A partir de las entrevistas se generaron cinco categorías de análisis. Como ideario de salud emergieron tres grandes dimensiones: física, psicológica y social; la dimensión ambiente no emergió. El ambiente está representado con connotaciones negativas. El ES delega la responsabilidad sobre el ambiente físico al estado y al mercado, mientras que el ambiente social como determinante de la salud de los niños es responsabilidad de la red de interacción más cercana al niño y si bien reconocen que el mercado influye en el ambiente alimentario, la responsabilidad final de la alimentación es considerada un hecho individual. CONCLUSIONES: El ES no incorpora al ambiente como parte del ideario de salud. Al indagar sobre su impacto en la salud de los niños, consideran que está mediado por su padres y cuidadores, excepto en contaminación ambiental, donde el foco está puesto en el rol del estado y el mercado.

Palabras claves: Representaciones Sociales, Equipo de Salud, Salud, Ambiente, Escolares.

\begin{abstract}
INTRODUCTION: Environmental health concerns certain aspects of human health that includes life quality and it is defined by environmental, physical, social and dietary conditions.

OBJECTIVE: Investigate the social representations (RS) about the impact of the environment on the health of schoolchildren in the healthcare team (HTC) of two municipal Primary Health Care Centers (PHCC), Córdoba 2019.

METHODOLOGICAL DESIGN: The investigation was carried out through a qualitative perspective based on the Grounded Theory, with an emergent design. Thirteen open interviews were made through the constant comparison method. Data was validated by investigator triangulation.
\end{abstract}


RESULTS: From the interviews, five categories of analysis were designed. From Health perspective, three main dimensions were identified: physics, psychology and social, with the exception of environmental dimension. The environment is represented with negative connotations and not as the necessary mean for the upbringing and development of human beings. The HCT delegates the responsibility of the physical environment to the Government and the market. Meanwhile, the social environment as a determining of children health is responsibility of the interactive connection near to the child. Even though HCT acknowledges the market influences on the dietary environment, the final responsibility of food consumption relies on the individual.

CONCLUSION: The HCT does not include the environment as part of Health perspective. While investigating about the environment's impact on children's health, the HCT considers that is controlled by their parents and caregivers, with the exception of environmental contamination, in which the Government and the market's role are to blame for.

Keywords: Social Representations, Healthcare team, Health, Environment, Scholars.

\section{Introducción}

Desde el paradigma ecosocial la salud se conceptualiza como estado de bienestar físico, psíquico y social, con capacidad de funcionamiento. Desde este paradigma, uno de los objetivos de la salud pública es analizar la forma en que se estructura la respuesta social de las condiciones de salud ${ }^{1,2}$. La teoría ecosocial recoge los aportes de las teorías ecológicas y sistémicas aplicadas a la salud, considerando tres elementos: a) lo ecológico, b) la influencia de la estructura social en la producción de la salud y la enfermedad y c) las interrelaciones entre los dos primeros. Según Álvarez-Castaño ${ }^{4,5}$, la perspectiva ecosocial enfatiza que los estilos de vida no son decisiones individuales, sino comportamientos influenciados por las oportunidades definidas por el medio social en que las personas viven. Esta perspectiva se considera como una de las tantas teorías para analizar los determinantes de la salud ${ }^{3-5}$.

Las Representaciones Sociales (RS) son una expresión del conocimiento de sentido común, "es un corpus organizado de conocimiento y una de las actividades psíquicas, gracias a las cuales las personas hacen inteligible la realidad física y social, se integran en un grupo o en una relación cotidiana de intercambios y liberan los poderes de su imaginación". Las RS permiten interpretar el curso de los acontecimientos y las actuaciones sociales, expresan las relaciones que las personas mantienen con el mundo y con los otros. Es fundamental investigar acerca de las RS del equipo de salud ya que son los que tienen acceso más rápido a la información científica, pero conservan representaciones del sentido común sobre el ambiente en el que se desarrollan los niños en etapa escolar y cómo éste influye en su salud, que pueden interferir en el proceso de atención ${ }^{6}$.

Es importante prestar especial atención a las representaciones sociales de quienes intervienen en todo el proceso salud, enfermedad y atención
(PSEA); ellos pueden favorecer las distintas estrategias en la mejora general del estado sanitario de la población mediante actividades de Atención Primaria de Salud (APS) estableciendo un modo específico de comprender y resolver situaciones desde su punto de vista. Es necesario sensibilizar acerca de incluir de manera integral al ambiente en los aspectos relacionados a la atención en salud mediante una política de recursos humanos que permita las capacitaciones $\operatorname{adecuadas}^{7,8}$.

En Argentina el sistema público de salud está integrado por los hospitales públicos y los centros de atención primaria de la salud (CAPS) que funcionan bajo la coordinación de Ministerios y Secretarías de la Salud de las diferentes jurisdicciones (nacional, provincial o municipal) y presta servicios de provisión gratuita. Los CAPS fueron propuestos por la OPS como una manera de aplicar la estrategia de atención primaria de la salud y cumplir con los principios y objetivos de la meta de Salud para todos 9 . Para cumplir esta misión, un CAPS, debe tener un conocimiento preciso de las necesidades de la población y los recursos disponibles, con el fin de definir las acciones necesarias para responder a las realidades locales de salud ${ }^{9}$.

Las decisiones cotidianas que los equipos de salud toman sobre la atención de la salud de su población a cargo, no se restringen a decisiones sobre el conocimiento biológico de los procesos de salud enfermedad, sino que tiene relación con procesos más amplios de producción social. Una serie de circunstancias asociadas a la trayectoria social, a la ocupación, a la sociabilidad, otorga a las personas interacciones que retroalimentan las perspectivas personales sobre la atención de la salud. Estas interacciones están imbricadas en las propias representaciones que cada uno tenga acerca de la salud en general y del ambiente en particular ${ }^{10}$. 


\section{Objetivo}

Por lo antedicho se propone indagar las representaciones sociales (RS) del equipo de salud (ES) de dos Centros de Atención Primaria de Salud municipales (CAPS) sobre el impacto del ambiente en la salud de los escolares, durante el ciclo 2019 en la ciudad de Córdoba.

\section{Población y métodos}

El presente estudio se desarrolló en dos Centros de Atención Primaria de Salud de la Municipalidad de Córdoba, durante el año 2019; como parte del proyecto de investigación "Salud de escolares y ambiente en Córdoba: análisis desde un enfoque metodológico mixto", el cual fue aprobado y subsidiado por la Secretaria de Investigación de la Universidad Católica de Córdoba.

Se realizó un estudio desde la perspectiva interpretativa, basado en la teoría fundamentada (TF) con diseño emergente. La metodología propuesta por la teoría fundamentada se basa en dos grandes estrategias: el método de comparación constante y el muestreo teórico. A través del método de la comparación constante se recogió, codificó y analizaron los datos de forma simultánea, para generar teoría. Por otro lado, el muestreo teórico se realizó para descubrir categorías y sus propiedades, y para sugerir las interrelaciones dentro de una teoría ${ }^{11-13}$.

La población de estudio estuvo conformada por los trabajadores de salud que se desempeñan en dos CAPS municipales de la ciudad de Córdoba, en el año 2019; los individuos fueron seleccionados dependiendo de las expectativas que nos generaban de aportación de nuevas ideas, hasta lograr la saturación teórica.

La variable que compuso esta investigación fue las RS sobre el impacto del ambiente en la salud de los escolares. Esta variable fue analizada mediante una codificación abierta y selectiva con la posterior creación de categorías. Estas categorías surgieron a partir de las dimensiones de ambiente alimentario, físico y social; y, a partir de ellas, se crearon preguntas que pudieran responder cada categoría.

Como técnica se utilizaron entrevistas abiertas, se utilizó una guía de preguntas y se grabó la entrevista, siempre que el entrevistado prestó su consentimiento.

Para la realización del análisis de datos se implementó el método comparativo constante. Se llevó a cabo un análisis cualitativo mediante la elaboración de códigos o etiquetas con fragmentos de texto de las entrevistas que luego fueron relacionados y clasificados. La codificación supuso un corte o descontextualización de los datos. Por otro lado, admitió recuperarla en un nuevo texto (recontextualización) para descubrir sus propiedades y dimensiones. Para esto, fue necesario la transcripción de las entrevistas y la realización de una base de datos con los documentos primarios. La delimitación de la teoría se basó en: maximizar las similitudes y minimizar las diferencias, creando una categoría central.

Se utilizó un software, basado en la teoría fundamentada, llamado Atlas.Ti. Este es un programa de análisis cualitativo asistido por computadora (QDA)

Para asegurar la validez y fiabilidad de los datos, se utilizó la triangulación de investigadores, que consiste en la observación y posterior intercambio respecto a las entrevistas entre las autoras del trabajo; para reelaborar categorías consensuadas y afinar las interpretaciones acerca de $\operatorname{los}$ datos ${ }^{14}$.

\section{Consideraciones éticas}

El presente trabajo forma parte del proyecto de investigación "Salud de escolares y ambiente en Córdoba: análisis desde un enfoque metodológico mixto", el cual fue aprobado por el CIEIS Reina Fabiola. A su vez, fueron respetadas las recomendaciones de la Declaración de Helsinki y sus diferentes enmiendas.

\section{Resultados}

Acerca de los profesionales de salud dadores de cuidado:

Se realizaron 13 entrevistas abiertas, (11 profesionales y 2 no profesionales, administrativos). La (Tabla 1) muestra algunas características de los entrevistados.

La indagación de las representaciones en este trabajo se centró en la teoría fundamentada con diseño emergente, basándose en el método de comparación constante.

\section{Representaciones de Salud:}

Lo recabado en las entrevistas abiertas, se pudo identificar que gran parte de los profesionales definen la salud como bienestar:

... "El bienestar físico, emocional, espiritual; el estar bien. El poder encontrar un equilibrio de algún modo en la vida y sentirse feliz, bien" ... (Entrevista $\mathrm{N}^{\circ} 1, \mathrm{CS} \mathrm{N}^{\circ} 1$ )

Si bien la mayoría tiene en sus discursos este concepto de salud ampliado, dos entrevistados se 
refirieron a la salud como un concepto meramente preventivo:

...” La salud sobre todo de los niños depende de los controles de salud, de las vacunas, de que los papas los traigan para acá" ... (Entrevista $\left.\mathrm{N}^{\circ} 4, \mathrm{CS} \mathrm{N}^{\circ} 1\right)$

" Lo principal de la salud es la prevención porque si no se complica cuando traes los chicos no les haces los controles, después son cuadros más complicados, son más difíciles de resolver. Por eso están estos centros de AP” ... (Entrevista $\mathrm{N}^{\circ} 13$, CS $\mathrm{N}^{\circ} 2$ )

En las últimas dos entrevistas se establece un concepto más acotado del concepto de salud, muy ligado a su vivencia en el trabajo, teniendo en cuenta las acciones que se deben llevar a cabo con la finalidad de prevenir la aparición de enfermedades y lograr mantener un buen estado de salud. Otros profesionales, pudieron visualizar a la salud de manera más amplia, teniendo en cuenta las realidades de las personas, el bienestar subjetivo, objetivo y externo.

\section{Responsabilidad del Cuidado:}

$\mathrm{Al}$ indagar sobre los responsables de la salud de los niños, casi en su totalidad, los entrevistados expresaron que esta depende exclusivamente del cuidado de sus padres o criadores, fundamentando esta postura en el nivel educativo de éstos y de las capacidades que tienen para implementar distintas herramientas a la hora de la toma de decisiones en cuestiones de salud:

..." Fundamentalmente depende de los criadores. No voy a decir padres porque ahora está el termino ese, "criadores". Pasa que algunos niños no son criados por sus padres biológicos, son criados por sus abuelas, abuelos, por alguien que lo adoptó también" ... (Entrevista $\mathrm{N}^{\circ} 1, \mathrm{CS} \mathrm{N}^{\circ} 1$ )

..." Para mí de la familia... Primero y principal es la familia porque si vos tenes padres responsables, padres que se interesan digamos por la salud de sus hijos" ... (Entrevista $\mathrm{N}^{\circ} 3, \mathrm{CS} \mathrm{N}^{\circ} 2$ )

\section{Ambiente y Salud:}

En la relación entre ambiente y salud, se observa en los discursos la impronta de los contaminantes ambientales; es decir, saca el foco de la salud en positivo, para entender que el ambiente puede impactar negativamente en la salud:

..." Un ambiente puede ser tóxico, si yo estoy en un lugar de trabajo que para mí la gente laboral es tóxica, va a repercutir en mi salud" ... (Entrevista $\mathrm{N}^{\circ} 3$, CS No2)

." Influye porque es parte de la tríada Agente, Huésped y Ambiente" ... (Entrevista N 6 , CS N 1 )

En los discursos los entrevistados se delega la responsabilidad del cuidado del medio ambiente al estado y al mercado, mientras que describen al ambiente social como el entorno más cercano al niño:

... "El ambiente social empieza en su relación con su entorno pequeño-familiar" ... ... "los tenemos en nuestras manos y de ahí en adelante todos los que se relacionen con él lo pueden perjudicar mucho. Lo más importante es su alma, su cerebro y sus cuidados" ... (Entrevista $\mathrm{N}^{\circ} 7, \mathrm{CSN}^{\circ} 2$ )

Se observa que la mayoría de los integrantes del equipo de salud concibe al ambiente alimentario como el que rodea el acto de comer y que está determinado por su entorno más cercano:

...” Todo lo relacionado con la alimentación, el consumo, las creencias y mitos sobre la alimentación, lo que se transmite, las costumbres" ... (Entrevista $\left.\mathrm{N}^{\circ} 6, \mathrm{CS} \mathrm{N}^{\circ} 1\right)$

La (Figura 1) muestra la red conceptual de las categorías que emergieron en las entrevistas.

\section{Discusión}

Los resultados de este estudio coinciden con trabajos realizados por el grupo de investigación en Centros de Salud provinciales, en el que los profesionales coincidieron en una definición de salud desde el modelo de los determinantes de la salud, considerando el estar sano como una situación compleja e integral y que entenderla desde otro punto de vista es reduccionista ${ }^{15}$.

Coincidiendo con esta investigación, Andrade y $\mathrm{col}^{16}$ destaca que el medio ambiente y la salud aún se entienden como temas independientes, lo que dificulta acciones más eficientes por parte de la población y de las entidades gubernamentales y no gubernamentales.

En el presente estudio se pudo observar que el equipo de salud delega la responsabilidad sobre el medio ambiente al estado y al mercado, no siendo consciente sobre el impacto que tiene su propio accionar en él.

Aquí se pone en evidencia como único responsable del cuidado del ambiente al estado y coincide con Butze Aguilar ${ }^{17}$, que indica al estado como único árbitro, teniendo la obligación de dirimir los conflictos de intereses que se presentan entre la economía y el medio ambiente. Debido al 
imparable incremento de la actividad económica, la aceleración en la creación de necesidades, los modelos de consumo nuevos y la intensificación de la competencia por los mercados que demandan productos a un precio accesible $\mathrm{y}$, por ende, una producción a menor costo; conducen al deterioro del medio ambiente. Es por esto que el estado es un agente que debe inducir al mercado a que actúe de modo tal que se minimice el deterioro ambiental, o que se maximicen los efectos ambientales positivos de sus acciones. Por otro lado, en el último tiempo se ha conformado una corriente de pensamiento en donde se justifica la restricción de la libertad de aquellos que originan los problemas ambientales, con el objeto de proteger la libertad y el bienestar de los afectados. Tal restricción, se debe llevar a cabo por medio de la regulación directa, mediante normas impuestas por el estado con el objetivo de arribar a un resultado colectivo que la misma sociedad prefiere y que no puede obtener de manera individual ${ }^{17}$.

Según las RS del equipo de salud sobre el impacto del ambiente social en la salud, se puede destacar que la responsabilidad de cubrir y responder a las necesidades y problemas de la infancia en cuanto a salud es atribuida fundamentalmente a la red de interacción más cercana al niño, sus padres o cuidadores. Estos resultados no coinciden con lo que se investigó a las RS del derecho a la salud de sus cuidadores en el año 2016, en Córdoba, donde el ES apreció el rol del Estado para la garantía y cumplimiento de los derechos del niño ${ }^{18}$.

En cuanto al ambiente alimentario, el equipo de salud considera que la alimentación está influenciada principalmente por las creencias, usos y costumbres que mantienen las personas en una determinada sociedad y cultura. Además, se observó que el equipo de salud considera que el Mercado es un factor que influye a la hora de la selección y compra de alimentos, pero que la responsabilidad final de la alimentación es un hecho individual. Al igual que la salud de los niños es visibilizada como un asunto privado.

Figueroa Pedraza y Dixis ${ }^{19}$, concluyeron que el acceso a los alimentos requiere de aumentos nominales de los salarios -por la transferencia de aumentos de productividad o de reducciones en los márgenes de lucro- y del abaratamiento de los bienes-salario, en especial de los alimentos. El aumento de la renta real y, sobre todo, la reducción del peso relativo de los costos de la alimentación, son componentes indispensables en la constitución de un mercado interno de masas y a la igualdad social $^{19}$. Por el contrario, en este estudio, el equipo de salud considera que el acceso a los alimentos es responsabilidad de las familias.

\section{Conclusiones}

A partir de las entrevistas emergieron tres grandes dimensiones vinculadas a la salud: física, psicológica y social; mientras que la dimensión de ambiente no emergió. Es decir que, si bien tiene una mirada amplia de la salud, no incorpora al ambiente como parte de la misma.

En cuanto al ambiente físico, se considera desde connotaciones negativas: aparece la contaminación ambiental y el ambiente como vector de enfermedades y no como el medio oportuno para el desarrollo integral de las personas. Tampoco emergieron en los discursos los efectos de las acciones individuales sobre el ambiente.

En cuanto al ambiente social, el ES lo definió como la red de interacción más cercana a los niños, considerando a la familia como la única responsable de velar por la salud de ellos, dejando en segundo plano las acciones del Estado en esta cuestión.

Las RS sobre ambiente alimentario afirman que éste es determinado por el contexto más cercano al niño, caracterizado por las costumbres, creencias y prácticas que se adquieren a través de la familia y la cultura. Si bien reconocen que el Mercado influye a la hora de la selección y compra de alimentos; la responsabilidad final de la alimentación es un hecho individual.

Se puede concluir, que cuando se piensa en salud, el ambiente no está presente en las representaciones sociales del equipo de salud, pero el impacto del ambiente en la salud de los niños aparece con connotaciones negativas, y con diferentes responsables del mismo según se trate del ambiente físico, donde los responsables de su degradación son el estado y el mercado, mientras que el ambiente social y alimentario es considerado una responsabilidad de las familias.

Los resultados obtenidos conforman una base para futuras investigaciones, se considera que la educación ambiental debería formar parte de la currícula en las carreras de salud, y de esta manera incorporar en su práctica diaria la mirada del ambiente como determinante de salud.

\section{Bibliografía}

1. Bulacio J, De Grandis S, Fernández R., Gomila, Sfaello I, at. Manual de Salud Ambiental Infantil. en. www.paho.org/chi/. [Online]; 2009 [cited 2019 abril 14).

2. Frenk, J. La salud de la población.: Hacia una nueva salud pública. Fondo de Cultura Económica, 2016. 
3. Becker GS. Human Capital: a theoretical and empirical analysis, with special reference to education. 3d. Edition. Chicago: The University of Chicago Press; 1993.

4. Álvarez-Castaño L. Los determinantes sociales y económicos de la salud. Asuntos teóricos y metodológicos implicados en el análisis. Rev. Gerenc. Polít. Salud. 2014; 13(27): 28-40. doi: http://dx.doi.org/10.11144/Javeriana.rgyps13 -27.dses

5. Álvarez-Castaño LS. Los determinantes sociales de la salud: más allá de los factores de riesgo. Rev. Gerenc. Polit. Salud. 2009; 8 (17):69-79

6. Mora M. La teoría de las representaciones sociales de Serge Moscovici. Athenea Digital. 2002; 2 .

7. Darget, M. Residencias Ministerio de Salud de la Provincia de Buenos Aires. El ambiente como determinante de la salud. Buenos Aires; 2009. (Dargett, MS. El ambiente como determinante de la salud. En IX Jornadas de investigación, docencia, extensión y ejercicio profesional:" Transformaciones sociales, políticas públicas y conflictos emergentes en la sociedad argentina contemporánea. La Plata, octubre 2014.

8. Moyca J, Palacios - Flores E, Medina A, Velásquez J, Castañeda D. Percepciones del Personal de Salud y la Comunidad sobre la adecuación cultural de los servicios materno perinatales en zonas rurales andinas y amazónicas de la región Huánuco. Revista Perú Med Exp Salud Pública. 2009; 26(2):145-160.

9. Paganini JM, Etchegoyen G, Arrondo F. Guía para el análisis y evaluación de los efectores de primer nivel. - 1a ed. - La Plata: Univ. Nacional de La Plata, 200931 p

10. Santillán L. Educación FLACSO ARGENTINA, Facultad Latinoamericana de Ciencias Sociales. Las iniciativas educativas familiares bajo análisis: notas sobre la dimensión social y política del cuidado infantil. 2012. [En línea]. Disponible en: http://www.propuestaeducativa.flacso.org.ar/ archivos/dossier_articulos/61.pdf

11. Ochoa D. La teoría fundamentada como metodología para la integración del análisis procesual y estructural en la investigación de las representaciones sociales. Revista CES Psicología. 2013;6(1):122-133.

12. Páramo Morales D. Teoría fundamentada, Metodología cualitativa de investigación científica. Revista Pensamiento \& gestión. 2015;(39):1-7.

13. Strauss A, Corbin J. Bases de la investigación cualitativa. Técnicas y procedimientos para desarrollar la teoría fundamentada. Colombia: Universidad de Antioquia; 2002. 354 p.

14. Pineda E., De Alvarado E., H. de Canales F. Metodología de la investigación. Manual para el desarrollo de personal de salud. $2^{\circ}$ edición. Washington, EUA. 1994. 39-47p.

15. Boni L, Crespo L. Estado nutricional de niños y representaciones sociales del derecho a la salud de sus cuidadores [Tesis de grado]. Córdoba: Universidad Católica de Córdoba. Facultad de Ciencias de la Salud; 2016.

16. Andrade J, Souza M, Brochier, J. Representación social de la educación ambiental y la educación sanitaria en estudiantes universitarios. Psicología: Reflexión y crítica, 2004. 17 (1), 43-50

17. Butze Aguilar W. Permisos de contaminación negociables: un instrumento de mercado para la regulación ambiental. Red de Revistas Científicas de América Latina, el Caribe, España y Portugal. 2006; 21(48): 255-288.

18. Prece G, Necchi S, Schufer M, Adamo M. La salud: una tarea cotidiana. Tercer SeminarioTaller de Investigación en Ciencias Sociales y Salud. Secretaría de Ciencia y Técnica, Ministerio de Educación y Justicia. 1988

19. Figueroa Pedraza D. Acceso a los alimentos como factor determinante de la seguridad alimentaria y nutricional y sus representaciones en Brasil. Revista Costarricense de Salud Pública. 2005; 14(27): 77-86. 


\section{Anexo tablas y figuras}

Tabla 1. Descripción de las características de los profesionales de salud cuidadores del niño.

\begin{tabular}{|c|c|c|c|c|c|c|}
\hline $\mathbf{N}^{\circ}$ & Profesión & Sexo & Edad & CS & Antigüedad & $\begin{array}{c}\text { Vive en } \\
\text { la zona }\end{array}$ \\
\hline 1 & Lic. en Psicología & F & 54 & 1 & 1 mes & no \\
\hline 2 & Lic. en Nutrición & F & 25 & 2 & 3 meses & no \\
\hline 3 & $\begin{array}{c}\text { Lic. en } \\
\text { Enfermería }\end{array}$ & F & 35 & 2 & 2 años & no \\
\hline 4 & Secretaria & F & 60 & 1 & 12 años & si \\
\hline 5 & Médica Clínica & F & 40 & 1 & 2,5 años & no \\
\hline 6 & Médica Pediatra & F & 42 & 1 & 12 años & no \\
\hline 7 & $\begin{array}{c}\text { Médica } \\
\text { Ginecóloga }\end{array}$ & F & 56 & 2 & 5 años & no \\
\hline 8 & $\begin{array}{c}\text { Lic. en } \\
\text { Enfermería }\end{array}$ & F & 52 & 1 & 12 años & no \\
\hline 9 & Médico Clínico & M & 47 & 2 & 15 años & si \\
\hline 10 & $\begin{array}{c}\text { Lic. en } \\
\text { Enfermería }\end{array}$ & F & 56 & 2 & 20 años & si \\
\hline 11 & Medica Pediatra & F & 53 & 1 & 19 años & no \\
\hline 12 & Médica Pediatra & F & 45 & 2 & 1,5 años & si \\
\hline 13 & Secretario & M & 50 & 2 & 1 año & no \\
\hline
\end{tabular}


Scruzzi G F, Rodríguez Junyent C, Tosini C, Bertaina C, Bustos M C. Impacto del ambiente en la salud escolar: representaciones sociales del equipo de salud.

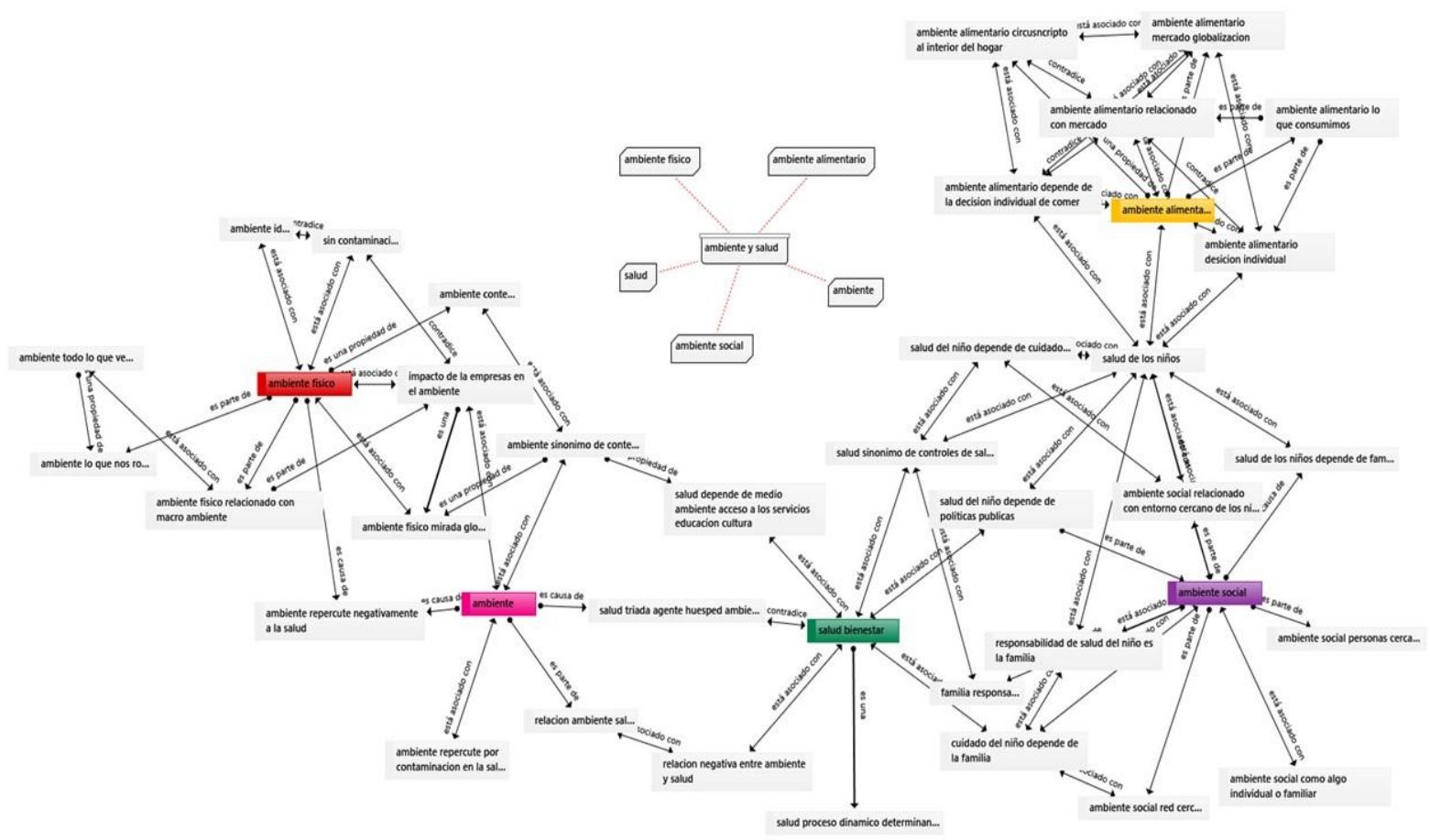

Figura 1. Red Conceptual del impacto del ambiente en la salud de los escolares, desde las representaciones sociales del equipo de salud de dos CAPS. Durante el ciclo 2019 en la ciudad de Córdoba.

\section{(c) (1)(8)

